moment can provide information on magnetic structures, and neutron wavelengths are comparable to atomic length scales. Neutron microscopy will find applications-for example, in morphology and crystallization studies - in probing materials from a variety of scientific and industrial fields.

RICHARD LOUIE

\section{Simulations Indicate Aluminum Has a Higher Ideal Shear Strength than Copper}

The theoretical shear stress or ideal shear stress is the stress necessary to deform a perfect crystal without defects. Usually this value is obtained through theoretical first principles-calculations, although in some instances nanoindentation techniques can serve as an experimental measurement of the ideal shear stress. In the case of aluminum and copper, a recent report of calculations based on density functional theory (DFT) showed that copper has a higher ideal shear strength than aluminum. However, Shigenobu Ogata, Ju Li, and Sidney Yip from the Massachusetts Institute of Technology have found the opposite result also from DFT calculations, as they report in the October 25 issue of Science.

After performing calculations using various DFT methods, results showed that aluminum has the higher ideal shear strength. The scientists followed the same procedure used to obtain the results previously reported, and also used additional methods that further corroborated their results. The researchers based their simulations on a six-atom supercell of three $\{111\}$ layers to calculate the equilibrium lattice constant and the relaxed and unrelaxed $\{111\}<11 \overline{2}>$ shear moduli. A 24-atom supercell of 12 layers for aluminum (10 for copper) served to calculate the intrinsic and unstable stacking-fault energies. The state of stresses considered for the calculations was pure shear or simple shear, with calculated stress values having an uncertainty of $<0.1 \mathrm{GPa}$.

The investigators attribute this result to the fact that the elastic strain of aluminum at the maximum shear stress is more than $50 \%$ larger than that of copper. The ideal pure shear strength of aluminum is $32 \%$ larger than that of copper, despite the fact that the latter has a $25 \%$ larger pure shear modulus along the $\{111\}<11 \overline{2}>$ system. The investigators also attribute this effect to the differences in ion relaxations in both materials: aluminum has almost no relaxation in the $x$ direction, and copper in the $\mathrm{z}$ direction. Therefore, stress applied in the $\langle 110\rangle$ direction hardens copper and softens aluminum, and stress applied in the $\langle 111\rangle$ direction has the opposite effect. As a consequence, interpretation of results from nanoindentation has to account for analysis of the state of stress, said the researchers. Aluminum atoms also have directional bonding which causes a longer shear deformation, higher intrinsic stackingfault energy, and higher ideal shear strength, they said.

SIARI S. SOSA

\section{Neutrons Used for Holographic Imaging of a Pb Crystal}

Electron and x-ray holography are well-known techniques for imaging the atomic structure of condensed materials. However, these methods are still limited because electrons interact strongly with matter, allowing only surface imaging as a practical matter, and $\mathrm{x}$-rays experience great variations of sensitivity for different atomic elements. Recently, L. Cser and Gy. Török from the Central Research Institute for Physics, Hungary, and C. Krexner from the University of Vienna, Austria, proposed experimental setups that enable holography imaging using thermal neutrons with wavelengths close to interatomic distances. The first approach, called an "inside-source concept," is based on large incoherent neutron scattering cross sections of nuclei such as hydrogen that could serve as pointlike sources of neutron spherical waves inside the sample. The second, called an "inside-detector concept," is based on the use of strongly neutronabsorbing isotopes acting as pointlike detectors in the sample.
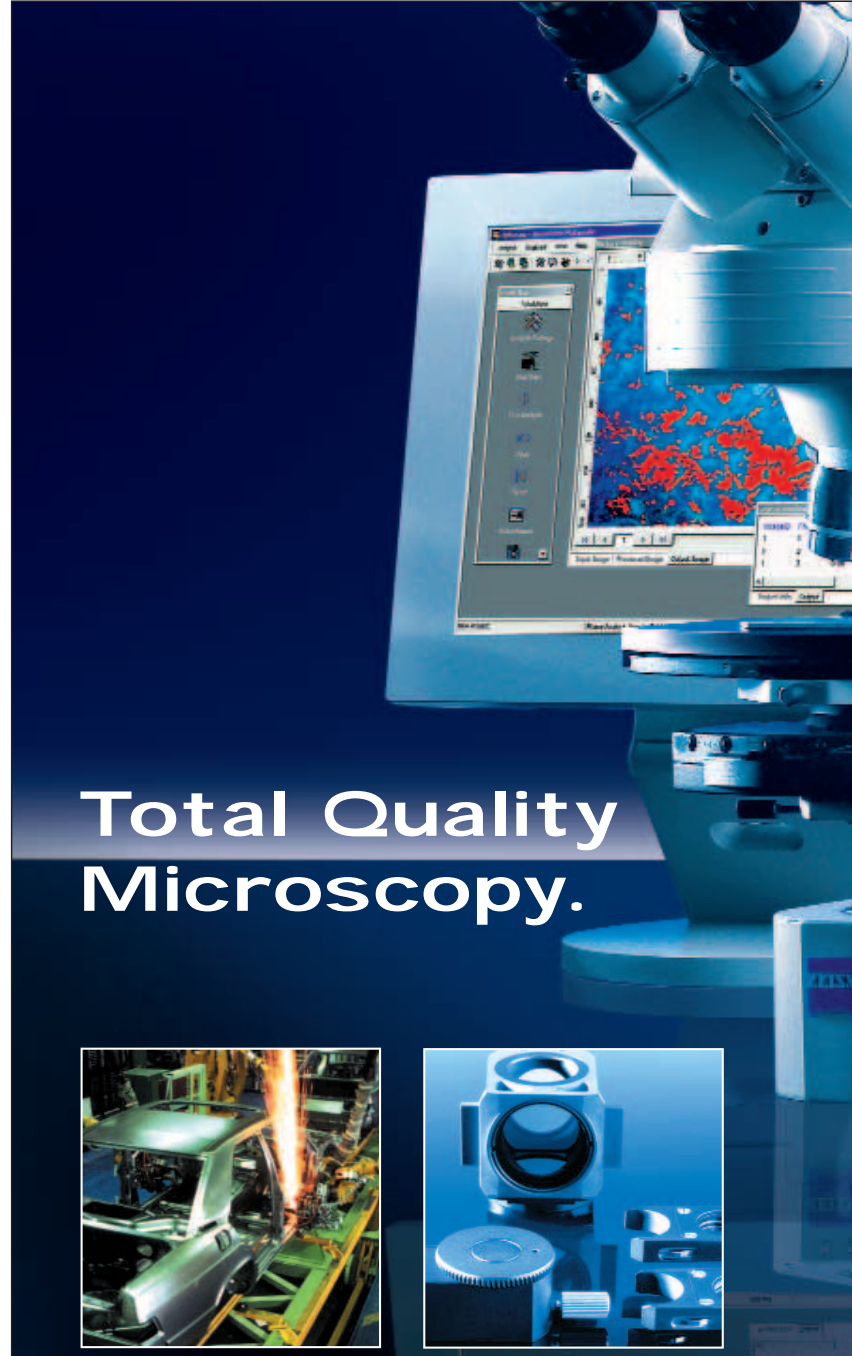

There is a new dynamic force in materials microscopy - advanced materials with increasingly complex properties are demanding new techniques and systems. Now an exciting world of integrated systems for materials microscopy is meeting the challenge of these dynamics - with outstanding materials microscopes, innovative techniques, high resolution digital cameras and superior imaging software. This world of systems is based on the Carl Zeiss philosophy: Total Quality Microscopy.

\section{Materials microscopy on the move.}

Carl Zeiss Microlmaging, Inc. Thornwood, NY 10594 1.800.233.2343 micro@zeiss.com zeiss.com/micro

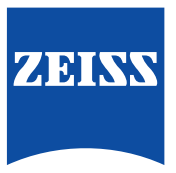

We make it visible. 
As reported in the October 21 issue of Physical Review Letters, the researchers in cooperation with I. Sharkov from St. Petersburg State University, Russia, and B. Farago from Institut Laue-Langevin, France, realized the inside-detector concept for neutron holography imaging of lead nuclei in a $\mathrm{Pb}(\mathrm{Cd})$ single crystal. The researchers used a spherical-shaped single crystal of $\mathrm{Pb}_{0.9974} \mathrm{Cd}_{0.0026}$ for this experiment. It is known that the $\mathrm{Cd}$ absorption cross section for thermal neutrons is more than four orders of magnitude larger than for $\mathrm{Pb}$, thus $\mathrm{Cd}$ atoms could act as highly efficient detectors. Due to the low concentration of $\mathrm{Cd}$, these atoms are randomly distributed on regular lattice sites and any one $\mathrm{Cd}$ atom is surrounded by $\mathrm{Pb}$ atoms. The lead nuclei play the role of the object while the cadmium nuclei serve as pointlike detectors inside the sample by emitting gamma rays that are detected outside the crystal.

The researchers registered the gammaray image and reconstructed the atomic structure by use of a computer conversion of a hologram to a three-dimensional picture. Furthermore, the researchers restored the object without making use of any a priori knowledge about the orientation of the sample.

The researchers said that their results demonstrate the feasibility of atomicresolution neutron holography as it was originally proposed, so the practical applications appear highly promising. They said that the special properties of the nuclear scattering process of neutrons, including its isotopic sensitivity and its dependence on the magnetic moment, will substantially enlarge the field of investigation opened by this technique.

$$
\text { ANDREI A. ELISEEV }
$$

\section{Coherent X-Ray Technique Reveals Interface Structure with Sub-Angstrom Resolution}

Several nondestructive methods are available to study the structure of epitaxial films and interfaces, but interpretation of the results depends on the mathematical model applied, which sometimes produces incorrect conclusions. As reported in the October issue of Nature Materials, a group of researchers have developed an x-ray method, called coherent Bragg rod analysis (COBRA), for the direct determination of epitaxial structures. Analyzing the coherent Bragg rods, Yizhak Yacoby and Mukhles Sowwan of Hebrew University, Israel, and their co-workers at the University of Washington, Seattle; Argonne National Laboratory, Illinois; Brookhaven National Laboratory, New York; and the University of Michigan, Ann Arbor, were able to map with atomic precision the structure of the interfacial region of a $\mathrm{Gd}_{2} \mathrm{O}_{3}$ film (2.7 $\mathrm{nm}$ thick) grown epitaxially on a (100)GaAs substrate.

A synchrotron beam line at the Advanced Photon Source was used for observing the diffraction pattern and the sharp Bragg peaks were detected together with the scattered intensity between peaks. COBRA, the technique used in this work, consists of analysis of intensity along the line between any two peaks in reciprocal space. The scattered intensity characterizes the two-dimensional periodic regions such as the substrate truncation, the interface, and the $\mathrm{Gd}_{2} \mathrm{O}_{3}$ film itself. The researchers measured 13 symmetrically nonequivalent Bragg rods and calculated the others using symmetry. With COBRA, the researchers were able to obtain the position of maximum electron density corresponding to the $\mathrm{Gd}$ in the unit cell of each layer of the film with a precision of $0.2 \AA$. The researchers found that for the $\mathrm{Gd}_{2} \mathrm{O}_{3}$ layers nearest the interface the position of the $\mathrm{Gd}$ atoms favored the stacking order of the GaAs substrate, whereas for layers further from the interface the stacking order relaxed to the value of bulk $\mathrm{Gd}_{2} \mathrm{O}_{3}$.

MAXIM NIKIFOROV

\section{Superconductivity Attained in Compressed Lithium}

Under close scrutiny in superconductivity are methods of raising the transition temperature to the superconducting state. It is believed, based on BCS theory, that highly compressed materials with low atomic numbers can yield transition temperatures near room temperature. Katsuya Shimizu's group from Osaka University and Takehiko Yagi from the University of Tokyo have demonstrated the ability to attain superconductivity under high pressure in lithium at temperatures of $20 \mathrm{~K}$. This is the highest transition temperature for any element and supports the hypothesis that low atomic number elements have high transition temperatures.

As reported in the October 10 issue of Nature, high-purity (99.999\%) lithium samples were exposed to pressures ranging from $3.5 \mathrm{GPa}$ to $48 \mathrm{GPa}$. All treatments were done in ambient argon atmosphere with no water or oxygen present. Pressures greater than $10 \mathrm{GPa}$ were applied at temperatures less than $50 \mathrm{~K}$ to control the effect of chemical reaction between lithium and the diamond anvil. Resistivity of the sample was monitored during the experiments. From the experimental results it has been demonstrated that a drop in the resistance occurs in samples compressed to more than $35 \mathrm{GPa}$. The transition temperature was determined to be $13 \mathrm{~K}$ at pressures of $35 \mathrm{GPa}$ and $20 \mathrm{~K}$ at pressures of $48 \mathrm{GPa}$. At lesser compressions the sample exhibited normal metallic behavior. After releasing the pressure, the sample exhibited its normal reflection of light, an indication of little or no chemical change during the process. Based on this, the researchers concluded that the change in resistance was due to the onset of superconductivity in lithium.

Lithium is the first pure element to exhibit elevated superconducting properties as demonstrated by Shimizu, Yagi, and colleagues. Based on theoretical calculations, superconducting phase-transition temperatures of $80 \mathrm{~K}$ should be attained by further increasing the sample compression up to values of $165 \mathrm{GPa}$ in the cI16 phase, that is, a cubic unit cell with 16 atoms. According to the researchers, "High-pressure investigation of Li... should help in the understanding both of fundamental properties of metals and of possible room-temperature superconductivity in metallic hydrogen."

Calin Miclaus

\section{Synchrotron Source X-Ray Diffraction Used to Study Phase Transformations in Steel}

Phase transformation kinetics are important during processing of polycrystalline metals, steels for example, and ceramics. The kinetics are governed by grain nucleation and growth which ultimately also dictate the mechanical properties of the material, depending on the final average grain size. Though there are a number of proposed phase transformation models, the kinetics are still not well understood. Most of these models are based on the classical nucleation theory (CNT) and the law of parabolic grain growth derived by Zener. Now, a multinational European research group led by S.E. Offerman of Delft University, The Netherlands, has used a synchrotron source to obtain x-ray diffraction data from steels to study transformation kinetics at the level of individual grains. The researchers' results are published in the November 1 issue of Science.

The principal transformation reaction in carbon steels is from the high temperature fcc austenite to the low-temperature bcc ferrite. The solubility of carbon in ferrite is lower than in austenite, resulting in the enrichment of carbon in the remaining austenite. At lower temperatures, the carbon-enriched austenite decomposes into pearlite, which is a lamellar structure of ferrite and orthorhombic cementite. In addition, other complex nonequilibrium structures such as bainite and martensite 